\title{
EDITORIAL
}

\section{Clustering in asthma: why, how and for how long?}

\author{
Arnaud Bourdin*,\# and Pascal Chanez ${ }^{\top,+}$
}

$\Delta$ sthma and most chronic airway diseases are heterogeneous entities. Even severe asthma does not represent a single phenotype of asthma. This heterogeneity of phenotypes is the starting point of new approaches for characterisation, understanding and management of asthma in the near future. The ultimate approach will be to identify new phenotypes sharing coherent underlying biological mechanisms (i.e. the concept of endotypes [1]) to better predict future risks. Ultimately, new specific, targeted or personalised therapeutic avenues and management will be developed based on these new groupings of patients and used [2].

The main challenge is to avoid pre-established hypotheses. An unbiased approach of phenotyping is appealing in view of these ambitious goals. The increased popularity of clusters analysis in asthma benefits from the development of cohorts of asthmatic patients worldwide [1, 3-5]. For example, clusters derived from the Severe Asthma Research Program (SARP) initiative in the USA have been well received and disseminated within the severe asthma community [4]. This phenomenon can be explained by different factors: the approach was found original, the expert centres involved were excellent, and the findings and statistics were of great value. The items chosen in the hierarchical tree accounted for $85 \%$ of the variance of the clusters. Despite important interest demonstrated by specialists, most physicians are unable to fully understand the clustering process, and its translation in the real world to treat severe asthma is not obvious. The hypothesis-driven clustering approach used in the UK [5] led the authors to propose a cluster-based specific management built on the coherence between eosinophilic inflammation based on cellular percentage in induced sputum, and inhaled corticosteroid (ICS)-dose adjustment. In some small subgroups of patients, they used their results to safely step down ICS in non-eosinophilic patients, mainly obese females with uncontrolled asthma. In eosinophilic asthma patients, the induced-sputum based ICSdose management improved asthma control, leading to a decrease in the numbers of severe asthma exacerbations.

Nevertheless, we expect the clustering approaches to anticipate future risks (exacerbation, decline in lung function) and try to translate personalised medicine into reality.

\footnotetext{
*Département de Pneumologie et Addictologie, Hôpital Arnaud de Villeneuve, CHU Montpellier, \# INSERM U1046, Université Montpellier 1 et 2, Hôpital Arnaud de Villeneuve, CHU Montpellier, 'Département des Maladies Respiratoires, AP-HM, Marseille, and +'INSERM CNRS U 1067 UMR7733, Aix Marseille Université, Marseille, France.

CORRESPONDENCE: A. Bourdin, Département de Pneumologie et Addictologie, Hôpital Arnaud de Villeneuve, CHU Montpellier, France. E-mail: a-bourdin@chu-montpellier.fr
}

Physicians' daily practice is a real phenotyping exercise. This solid experience raises suspicion towards non-human statistical methods intended to validate our daily expertise. Usually, clustering reduces data to their mean differences, which does not necessarily reflect reality, especially the complexity of biological data. Moreover, this method is limited by the quality of data implementation. Implementing complex data (clinical history and follow-up, patients' outcomes, imaging and complex biology such as -omics approaches) will potentially lead to one cluster for each single individual with his/her unique phenotype.

Other methods have been tested before Ward's clustering approach, such as the principal component analysis [6] and the varimax rotations. They led to meaningful reports but to date, none has really surpassed the others. Lastly, a holistic approach [7] is advocated under the "unsupervised unbiased" label to avoid the selection bias introduced by the a priori given definitions of the disease.

In this issue of the European Respiratory Journal, KIM et al. [8] successfully used a clustering method to reach a potential "Holy Grail". For this purpose, they gathered data derived from two Korean severe asthma cohorts for a total of more than 2500 patients. They described four phenotypes between both cohorts who shared very similar patterns. This manuscript is of interest as it reports, for the first time, a clustering approach in asthma arising from Asia. Asthma was defined based on a World Health Organization definition and the data were collected on different occasions, which strengthened the impact of the study.

The four clusters were mostly discriminated by a three-axis components' scattergram comprising forced expiratory volume in $1 \mathrm{~s}$ (FEV1), age of onset and smoking. The latter is not often reported in studies as smoking is usually considered as an exclusion criterion in asthma; however, smoking and asthma represents a frequent clinical challenge for clinicians [9]. Accordingly, this clustering approach may be more applicable to our daily practice. The four clusters are reproduced in two large cohorts, they are relevant, and efficiently discriminate patients based on their past history (duration of the disease without anti-inflammatory treatment) and clinical examination (body mass index, rhinitis). Longitudinal assessment was used to investigate lung function decline. The authors report that pre-bronchodilator FEV1 does not decline in the follow-up year in all the clusters.

A potential limitation of the present study is the choice of items which contribute to the clusters, including the absence of the consumption of healthcare resources, comorbid conditions, inflammation and, obviously, treatments. The change in 
treatment and management may affect the validity of the longitudinal findings. Furthermore, this is a supervised cluster analysis, which dampens the potential comparison with other cluster reports, such as SARP.

Lastly, the clustering approach is an elegant way to better understand asthma using cohort data collection, yet clinical use in a daily practice in a primary or secondary care setting remains a matter of debate.

As long as clinicians remain capable of seeing human beings as unique individuals the scientific substrate for personalised medicine will remain their responsibility. Otherwise, predictive medicine, supported by any statistical method, will irremediably lose its credibility: "back to the trees" [10].

\section{STATEMENT OF INTEREST}

None declared.

\section{REFERENCES}

1 Lötvall J, Akdis CA, Bacharier LB, et al. Asthma endotypes: a new approach to classification of disease entities within the asthma syndrome. J Allergy Clin Immunol 2011; 127: 355-360.
2 Bush A, Pedersen S, Hedlin G, et al. Pharmacological treatment of severe, therapy-resistant asthma in children: what can we learn from where? Eur Respir J 2011; 38: 947-958.

3 Just J, Gouvis-Echraghi R, Rouve S, et al. Two novel, severe asthma phenotypes identified during childhood using a clustering approach. Eur Respir J 2012; 40: 55-60.

4 Moore WC, Meyers DA, Wenzel SE, et al. Identification of asthma phenotypes using cluster analysis in the Severe Asthma Research Program. Am J Respir Crit Care Med 2010; 181: 315-323.

5 Haldar P, Pavord ID, Shaw DE, et al. Cluster analysis and clinical asthma phenotypes. Am J Respir Crit Care Med 2008; 178: 218-224.

6 Schatz M, Mosen D, Apter AJ, et al. Relationships among quality of life, severity, and control measures in asthma: an evaluation using factor analysis. J Allergy Clin Immunol 2005; 115: 1049-1055.

7 Bousquet J, Anto JM, Sterk PJ, et al. Systems medicine and integrated care to combat chronic noncommunicable diseases. Genome Med 2011; 3: 43.

8 Kim TB, Jang AS, Kwon HS, et al. Identification of asthma clusters in two independent Korean adult asthma cohorts. Eur Respir J 2013; 41: 1308-1314.

9 Polosa R, Thomson NC. Smoking and asthma: dangerous liaisons. Eur Respir I 2013; 41: 716-726.

10 Lewis R. The Evolution of Man: Or, How I Ate My Father. London, Vintage, 1994. 